\begin{tabular}{|l|l|}
\hline Postprint Version & 1.0 \\
\hline Journal website & $\underline{\text { http://www.sciencedirect.com/science/article/pii/S0738399111005532 }}$ \\
\hline Pubmed link & $\underline{\text { http://www.ncbi.nlm.nih.gov/pubmed/22079211 }}$ \\
\hline DOI & $10.1016 /$ j.pec.2011.10.002
\end{tabular}

This is a NIVEL certified Post Print, more info at http://www.nivel.eu

\title{
The importance of patient-centered care for various patient groups
}

DOLF DE BOER ${ }^{A, B}$, , DiANA DELNOIJ ${ }^{B},{ }^{c}$, JANY RADEMAKERS ${ }^{A}$

\footnotetext{
${ }^{a}$ NIVEL (Netherlands Institute for Health Services Research), Utrecht, The Netherlands

${ }^{b}$ CKZ (Centre for Consumer Experiences in Healthcare), Utrecht, The Netherlands

${ }^{c}$ Scientific Centre for Transformation in Care and Welfare (Tranzo), Tilburg University, Tilburg, The Netherlands
}

\begin{abstract}
Objectives: To assess differences in the importance ascribed to patient-centered care between various patient groups and demographic groups.

Methods: Survey data collected using questionnaires were analyzed for patients that underwent hip or knee surgery $(n=214)$, patients suffering from rheumatoid arthritis $(n=343)$, spinal disk herniation ( $n=145)$, breast abnormalities $(n=596)$ or congestive heart failure $(n=$ 118). A composite for patient-centered care priorities was constructed $(\alpha=0.82)$ and compared to the average importance over all healthcare aspects in the surveys.

Results: All patient groups considered patient-centered care to be of above-average importance ( $p$ ‘s $<0.001)$. Small but significant differences were observed: patient-centered care was more important for patients suffering from congestive heart failure $(p<0.001)$ and patients who were younger, female, well-educated and healthier ( $\left.p^{c} s<0.05\right)$. Patients who had undergone hip or knee surgery considered patient-centered care more important than patients with spinal disk herniation did $(p<0.05)$.

Conclusion: Patient-centered care is important to all patient groups. Differential policies regarding patient-centered care for patient subgroups do not seem required.

Practical implications: Given the importance attributed to patient-centered care, it is essential that elements of patient-centered care are included in surveys, indicators of quality of care, and the training of doctors and nurses.
\end{abstract}

\section{INTRODUCTION}

Patient-centered care is both a goal in itself and a tool for enhancing health outcomes [1]. For some time, it was generally assumed that patient-centered care is always superior to any other policy and more appreciated by patients as well. However, accumulating evidence suggests that not all patient groups are equally responsive to a patient-centered approach [2], [3], [4], [5] and [6]. Given that patient-centeredness has been identified as an important priority for improvement of healthcare [7], it is important to increase 
our understanding of the settings and circumstances that determine the extent to which patients appreciate patient-centered care.

Before discussing empirical literature on preferences for patient-centered care, the concept itself deserves some consideration, as a variety of interpretations and operationalizations have been adopted [2], [8], [9] and [10]. In their classic paper entitled 'Patient-centeredness, a conceptual framework and review of the empirical literature', Mead and Bower distinguished five dimensions of patient-centered care [8], all of which essentially materialize within the doctor-patient relationship. These dimensions are: the biopsychosocial perspective (i.e. the doctor also appreciates the psychological and social aspects of the health problem in addition to the biomedical aspects), the doctor as person (i.e. the doctor uses his/her own subjectivity and sensitivity as a tool to enhance the doctor-patient relationship), the patient as person (i.e. doctor is not just concerned with the disease but also attentive to the individual's personal circumstances and experience of illness), sharing power and responsibility (i.e. doctor involves the patient in decisionmaking and provides information to that end) and the therapeutic alliance (i.e. both a professional and personal partnership between patients and doctors that enhances the desired emotional context during consultations). It has been suggested that researchers should expressly state how they define patientcentered care. This will make systematic evaluation of the empirical literature [9] possible. We would therefore like to make clear that this study will employ a composite measure that focuses on the last three of the above-mentioned dimensions of patient-centered care. More specifically, we will address the following aspects of care as they are experienced by patients during the consultation: being listened to attentively, being able to ask questions, being taken seriously, receiving understandable explanations, and shared decision-making.

Although evidence looking at the extent to which patient experiences are reflected in their global ratings stresses the importance of these process aspects [11], several studies also suggest that patient-centered care is valued differently across demographic groups. It has been reported for example that patients have a higher preference for patient-centered care or elements of patient-centered care if they are female, white, younger, better educated, healthier or have a higher income [4], [5] and [6]. Similarly, Swenson et al. reported that younger patients and more highly educated patients appreciate a more patient-centered communication style [12]. In addition, a review by Janssen et al. showed a number of differences between younger and older cancer patients regarding the type of information and the amount of information they require [13]. Thus, there is evidence that demographic groups differ in the extent to which they value elements of patient-centered care.

Another way to categorize patients is to look at the health problem they present with. As far as the authors are aware, quantitative comparisons between patient groups with distinct health problems focusing on preferences for patient-centered care are limited. However, a number of studies indicate that the health problem at issue also partially determines whether patients prefer patient-centered care. For example, when asked which role individuals would prefer when it comes to decision-making regarding medical treatment, cancer patients are reported to prefer a significantly less active and responsible role compared to the general public [14]. In addition, cancer patients who recently experienced a substantial worsening of their condition are less likely to appreciate involvement in decision-making [15]. This finding is consistent with the more general notion that patients are less likely to engage in decision-making as disease severity increases [16]. Also, while in a patient-centered approach patients would be given the best information possible, cancer patients with a lower life expectancy are less likely to want to discuss life expectancy [17] and have also been reported to avoid certain information in an attempt to convey hope [18]. Further, a qualitative study from our group indicated that aspects regarding the information exchange between doctors and patients were scarcely included in the ten most important preferences of breast cancer patients, but were highly prevalent among the priorities of patients who underwent cataract surgery or hip or knee surgery [19]. Finally, when rating video vignettes of hypothetical consultations, participants' preferences for a directive or a shared approach appeared partially dependent on the health problem discussed [20]. Since in the latter study, participants were presented with a hypothetical situation, it remains to be determined whether there are differences between groups of patients who are actually suffering different medical problems.

Nevertheless, although fragmented, the available evidence more than justifies the hypothesis that the health problem at issue also partially determines the extent to which patients value patient-centered care.

Accordingly, it would be useful to compare the importance attributed to patient-centered care for a number of medical treatments and a number of demographic subgroups, where preferences are assessed using the 
same methodology. A family of questionnaires that is known as the Consumer Quality index (CQ-index or CQI) provides this opportunity.

The CQ-index is a Dutch instrument for measuring patient experiences. It is based on two other types of surveys: the American CAHPS (Consumer Assessment of Healthcare Providers and Systems) [21] and [22] and the Dutch QUOTE (quality of care through the patients' eyes) [23], [24], [25] and [26]. The CQ-index is characterized by its disease-specific and provider-specific focus as well as the assessment of patient priorities, which both derive from the QUOTE. The layout, response scales and standardized sampling, data collection, analysis, and presentation adopted for the CQ-index were taken from CAHPS. The CQ-index has been declared to be the national standard for measuring patient experiences and performance indicators of quality of care are frequently based on the CQ-index [27] and [28].

The present paper aims to explore further the potential differences in the importance of patient-centered care between patient groups with various health problems. Furthermore, differences between demographic groups will be examined. To that extent, data from the CQ-index were used on the following patient groups: hip or knee surgery, congestive heart failure, spinal disk herniation, rheumatoid arthritis, and malignant and benign breast abnormalities.

Research questions

(1) To what extent do patients from different patient groups value patient-centered care compared to the other healthcare aspects included in patient experience surveys?

(2) Are there any differences between patient groups in the importance they attribute to patientcentered care?

(3) Are there any differences between demographic subgroups in the importance they attribute to patient-centered care?

As it cannot be ruled out that potential differences between patient groups and demographic groups will be driven by the possibility that some patients rate everything as more important than others do, this possibility will also be examined in the present study.

\section{METHODS}

\subsection{Participants}

All data were collected in the Netherlands. Patients were identified through insurance companies and/or hospitals and approached by mail using a procedure known as the Dillman method [29], which includes up to four mail shots if necessary. The dataset for patients who underwent hip or knee surgery consisted of 214 patients (response $=78.6 \%$ ), the dataset for patients suffering from rheumatoid arthritis consisted of 343 patients (response $=59.6 \%$ ), the dataset for patients suffering from spinal disk herniation contained 145 patients (response $=37.1 \%$ ), the dataset for patients suffering from malignant and benign breast abnormalities consisted of 515 patients (response $=50.4 \%$ ), and the dataset for congestive heart failure consisted of 118 patients (response $=41.5 \%$ ). The aforementioned patient groups were selected because they represent a wide variety of conditions and because the surveys for these patient groups contained comparable items concerning patient-centered care. Data on the demographic characteristics (age, self-rated health, education and gender) are presented in Table 1 . The studies in which the data were collected were performed in accordance with the declaration of Helsinki.

\section{[TABLE 1]}

\subsection{Measurement of patient-centered care: construction of a composite measure}

For CQ-index surveys, the preferences of various patient groups are identified through focus group discussions that aim to identify important elements of care from the patient perspective that will be included in the survey [30]. Subsequently, the relative importance of each element is assessed using a questionnaire. The survey consists of a list of items for which patients may rate the importance. An example of a survey item could read 'To what extent do you think it is important that you are able to ask questions?' accompanied by four response categories (i.e. not important (1), of some importance (2), important (3), and 
extremely important (4)) [19]. For the present paper, five items from these importance surveys were selected to represent patient-centered care. Items on receiving understandable explanations and shared decision-making were taken to touch upon the 'sharing power and responsibility' dimension of patientcentered care. Being listened to attentively was taken to represent the 'patient as a person' dimension and being able to ask questions and being taken seriously were taken to refer to the therapeutic alliance dimension. Items focused on healthcare providing staff in general, rather than having separate items for doctors and nurses. Items were virtually identical between surveys except for the item 'receiving understandable explanations', which was not available in the survey for rheumatoid arthritis patients; for rheumatoid arthritis, an item concerning the importance of receiving understandable information was used instead. It is assumed that patients may interpret 'understandable information' to include written and/or verbal information, whereas 'understandable explanations' will be interpreted as verbal information only.

\subsection{Data analyses}

For each patient group, the items that represent patient-centered care appeared to form a reliable composite (Cronbach's $\alpha=0.82$, varying from 0.76 to 0.86 between patient groups) and accordingly, analyses were focused on this composite measure. Further, the average importance over all aspects included in the importance surveys - except those representing patient-centered care - was also calculated for each respondent. The number of aspects included in the average was 61 for congestive heart failure, 48 for hip or knee surgery, 63 for benign and malignant breast abnormalities and rheumatoid arthritis, and 68 for spinal disk herniation. The content of these aspects differed between surveys as they are based on focus group discussions with patients and stakeholder meetings [30]. Typical subjects covered in the surveys would be waiting times, activities to monitor disease, medication, accommodation, privacy, care following discharge from the hospital, etc. For each patient group, a paired samples t-test was used to assess whether the importance of patient-centered care differed significantly from the average importance. Cohen's $D$ is reported as an effect size for the difference between the average importance and the importance of patientcentered care. Generally, a Cohen's $D$ of 0.2 is considered a small effect, a Cohen's $D$ of 0.5 is considered a medium effect and a Cohen's $D$ of 0.8 or larger is considered a large effect. To assess the impact of patient group and demographic characteristics on the importance attributed to patient-centered care, a series of linear regression models were fitted. To account for the possibility that some patients rate everything as more important than other patients, the average importance was included as a covariate in several models.

\section{RESULTS}

\subsection{Importance of patient-centered care}

Table 2 shows the average importance score for all quality aspects that were included in the surveys containing importance items and the mean importance scores for the patient-centered care composite.

\section{[TABLE 2.]}

A series of paired samples $t$-tests demonstrated that for all patient groups, the importance of patientcentered care is significantly higher than the average importance score $(p<0.001)$. Table 2 shows that the sizes of the effects of the difference between the importance of patient-centered care and the average importance score are generally medium, except for patients suffering from a spinal disk herniation where the effect size was rather small (Cohen's $D=0.33$ ) and patients suffering from congestive heart failure where the effect size was large (Cohen's $D=0.90$; see Table 2).

\subsection{Differences between patient groups in the importance attributed to patient-centered care}

As can be seen in Table 2, the average importance over all items shows a similar pattern across patient groups compared to the importance of patient-centered care. Hence, it is possible that differences between patient groups in the importance attributed to patient-centered care are a result of some patients rating everything as more important than others. To examine this issue, we first fitted a linear regression model with the importance of patient-centered care as the dependent variable and the average importance as the 
independent variable $\left(F(1,1340)=1363.51 ; \mathrm{R}_{\text {adj }}^{2}=0: 50 ; ; p<0.001\right)$. Coefficients for all the regression analyses are reported in Table 3. Subsequently, the average importance was removed from the model and dummy variables indicating the patient group were entered $\left(F(5,1336)=13.03 ; \mathrm{R}_{\text {adj }}^{2}=0.043 ; p<0.001\right)$. Note that in accordance with Table 2 the importance of patient-centered care was the highest for patients with a breast abnormality (see Table 3). This model was then extended to include the average importance, yielding an explained variance that was approximately $1 \%$ higher than in the first model including only the average importance $\left(F(6,1335)=236.92 ; \mathrm{R}_{\text {adj }}^{2}=0.51 ; p<0.001\right)$. In this model, patient-centered care appears to be the most important for patients suffering from congestive heart failure (see Table 3 ). This is consistent with Table 2 as we are now essentially looking at the difference between the average importance and the importance of patient-centered care, which was the largest for congestive heart failure. Furthermore, it appears that patients who had undergone hip or knee surgery consider patient-centered care to be significantly more important than patients suffering from a spinal disk herniation do, as the confidence interval for hip or knee surgery (coefficient $\pm 2 \mathrm{SE}$ ) does not overlap with the coefficient for spinal disk herniation.

\section{[TABLE 3.]}

\subsection{Differences between demographic groups in importance of patient-centered care}

In order to assess whether the demographic characteristics age, sex, education and self-rated health may further contribute to the explained variance of the importance of patient-centered care, the model mentioned above was further extended to include these characteristics, yielding a $2 \%$ increase in the explained variance $\left(F(13,1328)=116.50 ; \mathrm{R}_{\text {adj }}^{2}=0: 53 ; p<0.001\right)$. The coefficients of this full model are presented in Table 3, which revealed that patient-centered care was considered to be significantly more important by patients who were younger than 65 , female, well educated and considered their health as excellent (see Table 3). Also note that this full model confirms our previous results regarding the effect of the average importance on the importance rating of patient-centered care and the differences in importance of patientcentered care between patient groups.

\section{DISCUSSION AND CONCLUSION}

\subsection{Discussion}

The present study demonstrated that - compared to all other healthcare aspects in the patient experience surveys - patients consider patient-centered care to be of above-average importance. This phenomenon is characterized by medium to large effects, except for patients suffering from a spinal disk herniation where the effect size appeared small. It appeared remarkably consistent across patient groups and is consistent with earlier research [11]. Furthermore, it was shown that the type of problem patients present with, as well as a number of demographic characteristics, predicted the importance that patients attribute to patientcentered care. However, both types of variables explained only a limited part of the variance in the importance attributed to patient-centered care. Importantly, these results were obtained while accounting for the possibility that some patients consider everything to be more important than other patients do, which appeared to have the most profound effect on the importance attributed to patient-centered care.

Consistent with previous research, patient-centered care was considered more important by patients who were younger, female, well educated and healthier [4], [5], [6] and [12]. As far as the authors are aware, this is the first demonstration that these effects appear to survive accounting for differences between patient groups as well as the possibility that some patients rate everything as more important than other patients. Admittedly, some previous studies required patients to make a tradeoff between various levels of decisional control, which prevents some participants rating everything as more important than others; results in terms of demographic characteristics were similar [14] and [31]. The impact of age, sex and educational level on the importance attributed to patient-centered care is therefore clearly a rather robust phenomenon. However, the variance accounted for was very restricted: approximately $2 \%$. Some previous studies appear to show 
somewhat more pronounced effects, but not to the extent that demographic variables were proposed as useful proxies of patient preferences for patient-centered care [4], [5], [6] and [14].

The present study showed that patients suffering from congestive heart failure consider patient-centered care to be more important than the other patient groups in our study. Furthermore, patients who underwent hip or knee surgery considered patient-centered care to be more important than patients suffering from a spinal disk herniation. Based on previous research, the observation that the importance of patient-centered care varies according to the health problem that patients present with was to be expected [14], [15], [19] and [20]. The present study expands on previous literature by providing an elaborate quantitative comparison of the importance of patient-centered care among a wide variety of patient groups using data collected with virtually identical methodology. Nevertheless, the differences observed between patient groups are limited and would not seem to support differential policies in terms of patient-centered care depending on the health problems that patients present with. Moreover, similarities between patient groups were much more striking than differences, with all patient groups rating the importance of patient-centered care as above average.

The observation that differences in preference for patient-centered care are limited at the group level (i.e. demographic groups or patient groups) does not preclude the existence of individual differences. These differences have been reported extensively [14] and [31] and it has even been shown that preferences change within individuals following a worsening of the disease [15]. As such, it has been suggested that doctors should exercise awareness regarding patient needs at each consultation [15]. As all patient groups in the present study regarded patient-centered care to be of above-average importance, doctors may choose to adopt a patient-centered approach as a default, but should also adapt their communication style in cases where it becomes clear during the consultation that a patient prefers otherwise.

Given the role of the measure of average importance in the present paper, this measure deserves careful consideration. The average importance was calculated as the average importance across all aspects in the surveys, excluding those incorporated in the patient-centered care composite. Typical health care aspects addressed in the surveys include waiting times, activities to monitor disease, medication, accommodation, privacy, care following discharge from the hospital, etc. The selection of health care aspects was based on focus group discussions with patients suffering from a specific health problem [30]. The aspects that patients considered important were selected for the survey, which was further refined through a stakeholder meeting. As such, the content of the surveys was allowed to differ between surveys, but the process by which these health care aspects were selected was standardized. We would argue that this is a desirable feature of the development of CQ-index surveys as it allows the specific needs of different health problems to be incorporated in the survey for that health problem. Nevertheless, it is important to keep in mind how the elements underlying the measure of average importance were selected when interpreting the present findings.

Accounting for the possibility that some patients rate everything as more important explained half of the variance in importance attributed to patient-centered care. It remains to be determined whether this phenomenon reflects a difference in response tendency between patients or whether some patients are just more concerned with (or involved in) the process of their health problem. Research on the different levels of patient activation suggests that the latter is the case and that real differences between patients do exist in this respect [32] and [33]. Nevertheless, researchers should seriously consider this issue when comparing patient groups or demographic groups in terms of the importance attributed to patient-centered care or any other aspect of healthcare.

\subsubsection{Limitations}

Some limitations regarding the present study should be considered. First, males were somewhat underrepresented in our results as a consequence of including data from patients who suffered from benign or malignant breast abnormalities. It is unlikely, though, that this limitation greatly affected our results as many of our findings are consistent across patient groups within the present study and/or consistent with previous research. Secondly, some of the response rates appeared low, particularly for patients suffering from congestive heart failure (respons $=41.5 \%$ ) or spinal disk herniation (response $=37.1 \%$ ). As such, it is possible that the results for these response groups are not entirely representative for the population from which they were drawn. Again, this limitation is probably limited as the surveys with rather low response rates show results that are consistent with those of the surveys with high response rates. Thirdly, the 
strategy by which importance was measured allowed some patients to rate everything as more important than other patients did. This was taken into account in our models, but can also be circumvented by an alternative method for measuring importance that requires respondents to perform a series of forced choices [34]. This has been done with similar results for the demographic characteristics [14] and [31], but it would also be worth verifying whether the present findings may be replicated using this alternative approach with regard to the effect of the health problem that patients present with. Finally, the way patient-centered care was measured deserves some consideration as it has been acknowledged that there is a lack of clarity in terms of the way researchers operationalize patient-centered, which hampers systematic research [9]. As indicated, patient-centered care is described using five dimensions [8]. Three of those dimensions were incorporated in the measure of patient-centered care in the present study: the patient as a person, shared power and responsibility and the therapeutic alliance. It should be conceded that each dimension was represented by only one or two survey items; these are undoubtedly not sufficient to capture the full complexity of each dimension. On the other hand, the combination of these items appeared to form a reliable composite for each patient group that provided a rather unique opportunity for quantitative comparisons between a wide variety of patient groups. On a similar note, it may be questioned whether it is appropriate to include different dimensions in a single measure. It has been acknowledged however, that the dimensions of patient-centered care may point in the same direction [9]. In addition, regardless of whether the dimensions of patient-centered care constitute different phenomena, the importance attributed to these phenomena may still be consistent within individuals, as indicated by the reliability of the patient-centered care composite in the present study.

\subsection{Conclusion}

In conclusion, the present paper clearly showed that patients consider the importance of patient-centered care as being above average, compared to other aspects of their care experience such as waiting times, activities to monitor disease, medication, accommodation, privacy, care following discharge from the hospital, etc. The importance attributed to patient-centered care differs between patient groups and demographic groups, but the magnitude of these differences appears limited.

\subsection{Practical implications}

Given the importance attributed to patient-centered care, it is essential that elements of patient-centered care are included in patient experience surveys, indicators of quality of care and the training of doctors and nurses. The present study would not seem to support differential policies with respect to patient-centered care for various patient groups or demographic groups, as the observed differences in preference between the groups were small. Nevertheless, individual differences in preferences for patient-centered care may still exist and, while a patient-centered approach might be a good start, doctors should be both perceptive and responsive to cues regarding the preferences of individual patients.

\section{ROLE OF FUNDING SOURCES}

The funding organizations played no role in the interpretation of the data, and in the preparation, review, or approval of the manuscript.

\section{CONFLICT OF INTEREST}

The author's declare they have no competing interests.

\section{ACKNOWLEDGEMENTS}

The authors would like to thank the organizations that funded the collection of the data. Zonmw, the Dutch organization for health research and development, funded the data collection for hip- or knee surgery, mammacare and rheumatoid arthritis. Zonmw and Miletus, an organization that represents health insurance companies, co-funded the data collection for spinal disc herniation. The data collection for 
Boer, D. de, Delnoij, D., Rademakers, J. The importance of patient-centered care for various patient groups Patient Education and Counseling: 2013, 90(3), 405-410

congestive heart failure was funded by Fonds PGO, a funding body for organizations representing patients and elderly or disabled individuals.

\section{REFERENCES}

[1] M. Meterko, S. Wright, H. Lin, E. Lowy and P.D. Cleary, Mortality among patients with acute myocardial infarction: the influences of patient-centered care and evidence-based medicine. Health Serv Res, 45 (2010), pp. 1188-1204.

[2] H. de Haes, Dilemmas in patient centeredness and shared decision making: a case for vulnerability. Patient Educ Couns, 62 (2006), pp. 291-298.

[3] E. Krupat, Patient-physician fit: an idea whose time has come. Med Decis Making, 26 (2006), pp. 110111.

[4] E. Krupat, J. Hsu, J. Irish, J.A. Schmittdiel and J. Selby, Matching patients and practitioners based on beliefs about care: results of a randomized controlled trial. Am J Manag Care, 10 (2004), pp. 814-822.

[5] E. Krupat, R.A. Bell, R.L. Kravitz, D. Thom and R. Azari, When physicians and patients think alike: patient-centered beliefs and their impact on satisfaction and trust. J Fam Pract, 50 (2001), pp. 10571062.

[6] E. Krupat, S.L. Rosenkranz, C.M. Yeager, K. Barnard, S.M. Putnam and T.S. Inui, The practice orientations of physicians and patients: the effect of doctor-patient congruence on satisfaction. Patient Educ Couns, 39 (2000), pp. 49-59.

[7] W. Richardson, D. Berwick, J. Bisgard, L. Bristow, C. Buck and C. Cassel, et al. Crossing the quality chasm: a new health system for the 21st century. Natl Acad Sci, (2001).

[8] N. Mead and P. Bower, Patient-centredness: a conceptual framework and review of the empirical literature. Soc Sci Med, 51 (2000), pp. 1087-1110.

[9] J. Bensing, Bridging the gap. The separate worlds of evidence-based medicine and patient-centered medicine. Patient Educ Couns, 39 (2000), pp. 17-25.

[10] N. Mead, P. Bower and M. Hann, The impact of general practitioners' patient-centredness on patients' post-consultation satisfaction and enablement. Soc Sci Med, 55 (2002), pp. 283-299.

[11] J. Rademakers, D. Delnoij and D. de Boer, Structure, process or outcome: which contributes most to patients'overall assessment of health care quality?. BMJ Qual and Saf, 20 (2011), pp. 326-331.

[12] S.L. Swenson, S. Buell, P. Zettler, M. White, D.C. Ruston and B. Lo, Patient-centered communication: do patients really prefer it?. J Gen Intern Med, 19 (2004), pp. 1069-1079.

[13] J. Jansen, J. van Weert, S. van Dulmen, T. Heeren and J. Bensing, Patient education about treatment in cancer care: an overview of the literature on older patients' needs. Cancer Nurs, 30 (2007), pp. 251260.

[14] L.F. Degner and J.A. Sloan, Decision making during serious illness: what role do patients really want to play?. J Clin Epidemiol, 45 (1992), pp. 941-950.

[15] P.N. Butow, M. Maclean, S.M. Dunn, M.H. Tattersall and M.J. Boyer, The dynamics of change: cancer patients' preferences for information, involvement and support. Ann Oncol, 8 (1997), pp. 857-863.

[16] J. Benbassat, D. Pilpel and M. Tidhar, Patients' preferences for participation in clinical decision making: a review of published surveys. Behav Med, 24 (1998), pp. 81-88.

[17] R.G. Hagerty, P.N. Butow, P.A. Ellis, E.A. Lobb, S. Pendlebury and N. Leighl, et al. Cancer patient preferences for communication of prognosis in the metastatic setting. J Clin Oncol, 22 (2004), pp. 17211730.

[18] G.M. Leydon, M. Boulton, C. Moynihan, A. Jones, J. Mossman and M. Boudioni, et al. Cancer patients' information needs and information seeking behaviour: in depth interview study. Brit Med J, 320 (2000), pp. 909-913.

[19] D. de Boer, D. Delnoij and J. Rademakers, Do patient experiences on priority aspects of health care predict their global rating of quality of care? A study in five patient groups. Health Expect, 13 (2010), pp. 285-297.

[20] B. McKinstry, Do patients wish to be involved in decision making in the consultation? A cross sectional survey with video vignettes. Br Med J, 321 (2000), pp. 867-871.

[21] J.L. Hargraves, R.D. Hays and P.D. Cleary, Psychometric properties of the Consumer Assessment of Health Plans Study (CAHPS) 2.0 adult core survey. Health Serv Res, 38 (2003), pp. 1509-1527.

[22] K.L. Carman, P.F. Short, D.O. Farley, J.A. Schnaier, D.B. Elliott and P.M. Gallagher, Epilogue: early lessons from CAHPS Demonstrations and Evaluations Consumer Assessment of Health Plans Study. Med Care, 37 (1999), pp. MS97-MS105.

[23] C.F. Hekkink, H.J. Sixma, L. Wigersma, C.J. Yzermans, J.T. Van Der Meer and P.J. Bindels, et al. QUOTE-HIV: an instrument for assessing quality of HIV care from the patients' perspective. Qual Saf Health Care, 12 (2003), pp. 188-193. 
Boer, D. de, Delnoij, D., Rademakers, J. The importance of patient-centered care for various patient groups Patient Education and Counseling: 2013, 90(3), 405-410

[24] M.D. Nijkamp, H.J. Sixma, H. Afman, F. Hiddema, S.A. Koopmans and B.B. van den, et al. Quality of care from the perspective of the cataract patient QUOTE cataract questionnaire. J Cataract Refract Surg, 28 (2002), pp. 1924-1931.

[25] H.J. Sixma, J.J. Kerssens, C.V. Campen and L. Peters, Quality of care from the patients' perspective: from theoretical concept to a new measuring instrument. Health Expect, 1 (1998), pp. 82-95.

[26] C. van Campen, H.J. Sixma, J.J. Kerssens, L. Peters and J.J. Rasker, Assessing patients' priorities and perceptions of the quality of health care: the development of the QUOTE-Rheumatic-Patients instrument. Br J Rheumatol, 37 (1998), pp. 362-368.

[27] M. Triemstra, S. Winters, R.B. Kool and T.A. Wiegers, Measuring client experiences in long-term care in The Netherlands: a pilot study with the Consumer Quality Index Long-term Care. BMC Health Serv Res, 10 (2010), p. 95.

[28] G.P. Westert, M.J. Berg van den, S.L.N. Zwakhals, J.D. Jong de and H. Verkleij, Dutch health care performance report, National Institute for Public Health and the Environment, Bilthoven, The Netherlands (2010).

[29] D.A. Dillman, Mail and Internet surveys: the tailored design method, John Wiley \& Sons Inc, New York (2000).

[30] O.C. Damman, M. Hendriks and H.J. Sixma, Towards more patient centred healthcare: a new Consumer Quality Index instrument to assess patients' experiences with breast care. Eur J Cancer, 45 (2009), pp. 1569-1577.

[31] T.F. Hack, L.F. Degner and D.G. Dyck, Relationship between preferences for decisional control and illness information among women with breast cancer: a quantitative and qualitative analysis. Soc Sci Med, 39 (1994), pp. 279-289.

[32] J.H. Hibbard and E.R. Mahoney, Toward a theory of patient and consumer activation. Patient Educ Couns, 78 (2010), pp. 377-381.

[33] K.J. Rask, D.C. Ziemer, S.A. Kohler, J.N. Hawley, F.J. Arinde and C. Barnes, Patient activation is associated with healthy behaviours and ease in managing diabetes in an indigenous population. Diabetes Educ, 35 (2009), p. 622.

[34] M. Ryan, Discrete choice experiments in health care. Br Med J, 328 (2004), pp. 360-361. 
TABLES

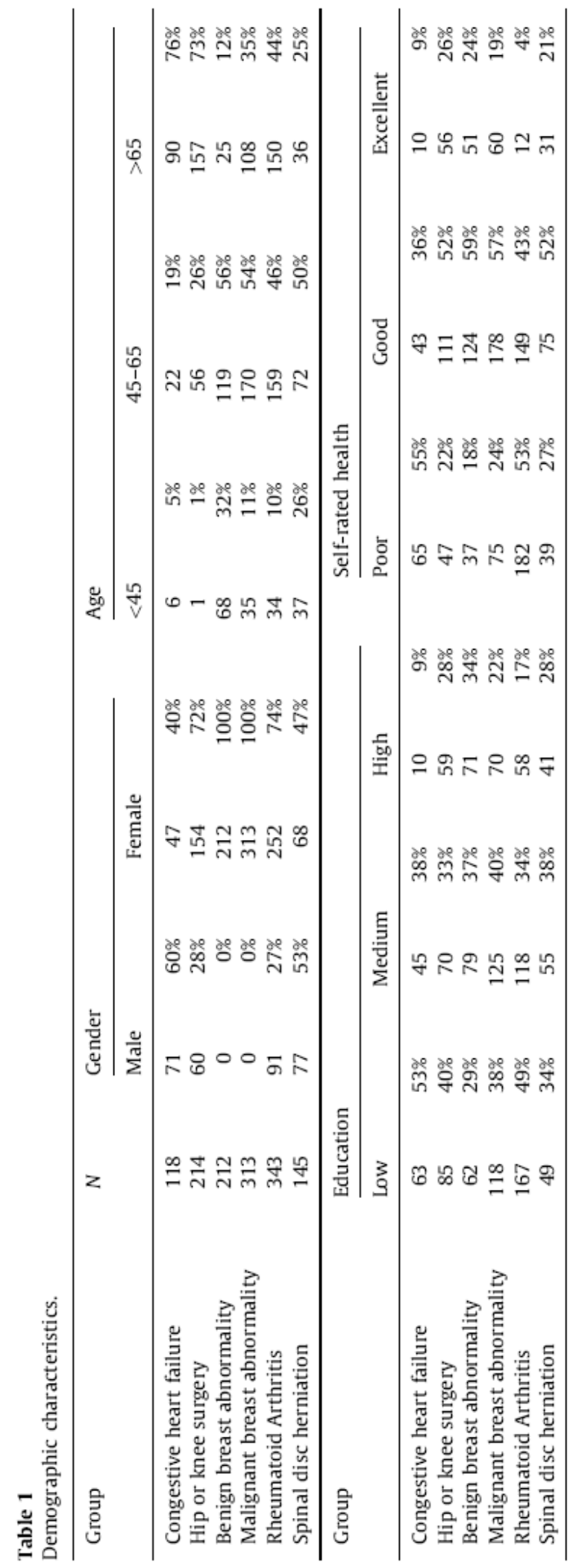




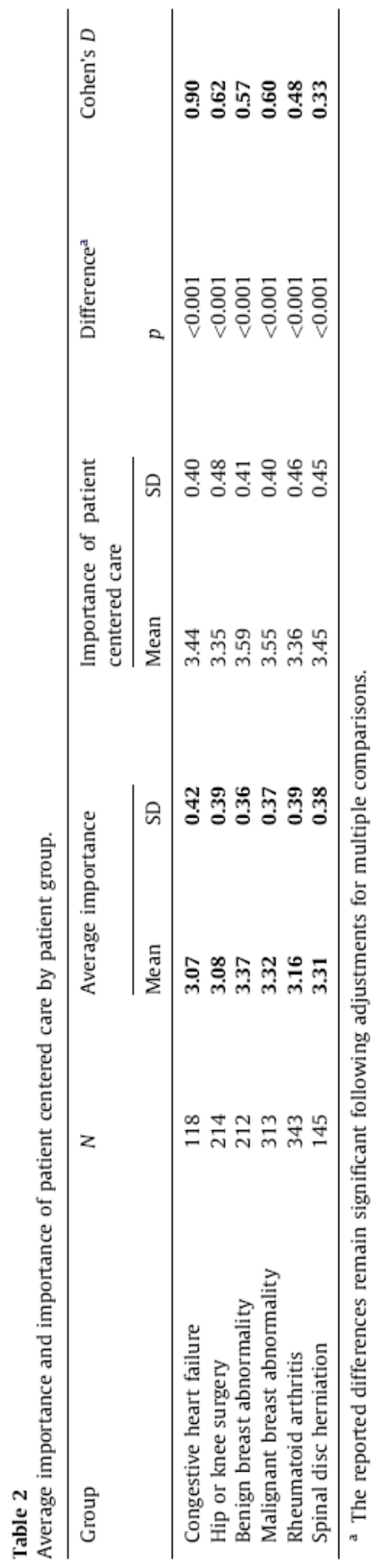




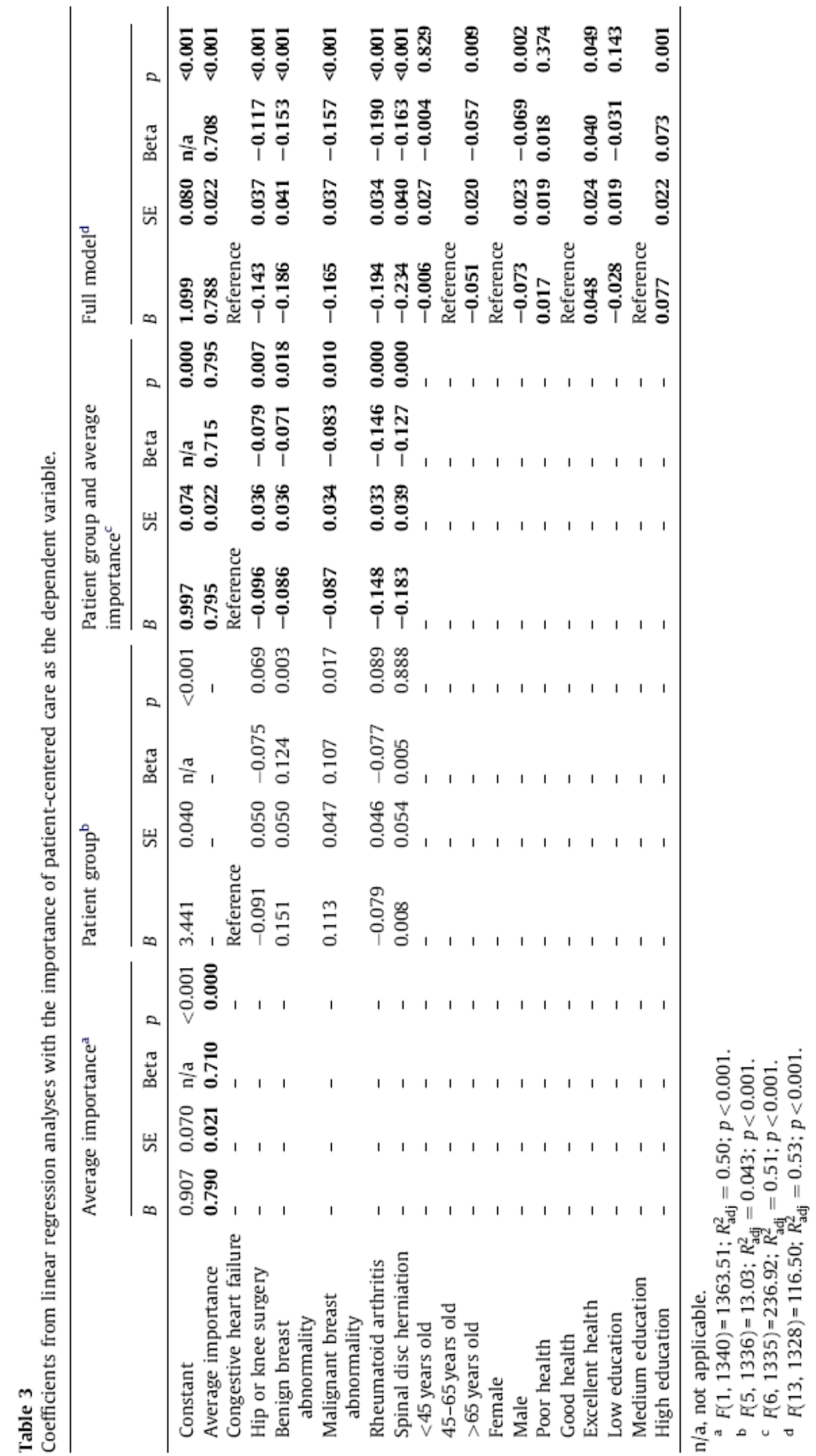

